\title{
Labyrinthe
}

16 | 2003

Afrique(s)

\section{Regards sur la décolonisation de l'Afrique Noire}

\section{Bernard Droz}

\section{OpenEdition}

Journals

Édition électronique

URL : http://journals.openedition.org/labyrinthe/306

DOI : 10.4000/labyrinthe.306

ISSN : 1950-6031

Éditeur

Hermann

Édition imprimée

Date de publication : 1 décembre 2003

Pagination : 9-18

Référence électronique

Bernard Droz, «Regards sur la décolonisation de l'Afrique Noire », Labyrinthe [En ligne], 16 | 2003, mis en ligne le 06 juin 2008, consulté le 30 avril 2019. URL : http://journals.openedition.org/labyrinthe/306 ; DOI : 10.4000/labyrinthe.306

Propriété intellectuelle 


\section{REGARDS SUR LA DÉCOLONISATION DE L'AFRIQUE NOIRE}

Bernard Droz

Les mille et un problèmes qui assaillent l'Afrique subsaharienne depuis une trentaine d'années - instabilité, sous-développement, corruption et violence, guerres intestines et inter-étatiques, interventions étrangères, migrations forcées, épidémies... - renvoient inévitablement aux conditions et aux modalités de son indépendance. Or, celle-ci avait fait l'objet, en son temps, d'une appréciation positive. Sans ignorer le chaos qui s'était installé dès la proclamation de l'indépendance au Congo belge, sans ignorer non plus les longues et cruelles guerres coloniales menées par le Portugal, on se plaisait à citer l'exemplarité d'un processus d'émancipation qui avait conduit les possessions anglaises et françaises à une indépendance librement négociée au terme d'une évolution politique fondée sur le dialogue et les perspectives d'une harmonieuse coopération. Étrangers à la tradition impérialiste de leur pays respectif, le général de Gaulle et Harold Macmillan étaient parvenus à la conclusion d'une nécessaire et rapide émancipation qui n'interdisait en rien la conclusion de liens préférentiels, les uns par la voie d'accords bilatéraux, les autres dans le cadre d'un Commonwealth élargi et rénové.

Sans être intégralement démenti, un tel optimisme a rapidement montré ses limites. Ni politiquement ni économiquement, les jeunes États africains n'ont su administrer les preuves de la maturité nécessaire à leur stabilité et à leur développement. Faut-il y voir un legs durable de l'infantilisme dans lequel la colonisation les a longtemps maintenus, ou le produit d'une incapacité structurelle à conduire leur avenir? Loin de tout tiers-mondisme, culpabilisateur des uns et hagiographique des autres, une rapide relecture de la décolonisation africaine doit pouvoir apporter quelques éléments de réponse.

L'éveil tardif de l'Afrique noire au nationalisme a été maintes fois relevé et peut-être est-ce dans la trop courte gestation/maturation de 
ce dernier qu'il faut comprendre son instabilité ultérieure. Rebelle au sentiment national en raison de sa très ancienne configuration tribale et de l'arbitraire du découpage ethnique qui a présidé à son partage, l'Afrique s'est vu imposer un ordre colonial qui reposait conjointement sur des chefferies dociles et sur le mirage de l'assimilation proposée à une mince élite, alors même que toute revendication d'émancipation sociale ou politique était impitoyablement réprimée. Pourtant, le développement du commerce et des infrastructures, le progrès de l'urbanisation ont ébauché une société détribalisée, de même que la mise en place d'un système scolaire, l'accession d'une minorité à l'enseignement secondaire et supérieur ont assuré la promotion d'une élite occidentalisée. Au même moment, les progrès de l'ethnologie sortaient de l'oubli le passé glorieux des États constitués de l'Afrique précoloniale et fondaient le concept de négritude qui traduisait l'ensemble de frustrations et de dépossessions de cette même élite. On ne saurait non plus négliger les échos d'un panafricanisme qui, dans le sillage de l'Américain Du Bois et du Jamaïcain Garvey, affirmait dès les années vingt la solidarité internationale du monde noir et ses droits à l'émancipation, ni l'introduction d'un marxisme simplifié diffusé par certains intellectuels comme George Padmore, qui a contribué à l'élaboration d'une idéologie populiste, à la fois nationaliste et socialisante, que tant de leaders africains ont adoptée par la suite.

Là où, dans le reste du monde colonisé, elle n'a eu qu'un effet d'accélération, la Seconde Guerre mondiale a revêtu en Afrique noire une importance décisive. À la différence de la guerre précédente, elle ne se limita pas à accroître la pression coloniale, à mobiliser les ressources et les hommes. Elle a eu pour effet d'accroître, surtout en Afrique occidentale, les activités de production, de transformation et d'échange et d'affirmer ainsi la promotion sociale des bourgeoisies urbaines. La capitulation belge, la défaite française, les difficultés britanniques démontraient les faiblesses et les divisions du pouvoir blanc, alors que les principes énoncés par la charte de l'Atlantique (1941) et celle des Nations unies (1945) - et particulièrement l'art. 73 de cette dernière, sous le titre de « Déclaration relative aux territoires non-autonomes » - recevaient un écho non négligeable en Gold Coast, au Kenya et à Madagascar.

Réunis à Manchester en mars 1945, quelque deux cents délégués d'Afrique anglaise réclament l'abandon du système conservateur et 
paternaliste de l'Indirect Rule et son remplacement par le Self-government. Faute de réponse appropriée - elle ne viendra, et timidement, qu'avec le Livre bleu de 1948 -, les leaders fraîchement sortis des universités anglaises (Nyerere, Kenyatta) ou américaines (Nkrumah, Azikiwe) prennent en main une mobilisation qui, sans revendiquer encore l'indépendance, dénonce sans relâche l'immobilisme administratif et les tares d'un système économique fonctionnant au profit quasi exclusif de la métropole et du colonat blanc. Sans négliger les rôles des syndicats, de la presse et de multiples associations culturelles, religieuses ou sportives, et même s'ils puisent souvent à des formations antérieures, les partis politiques, bien tenus par un leader incontesté, sont la conquête majeure de l'après-guerre. Fait nouveau, ils sortent du cadre restreint de l'intelligentsia ou de la classe moyenne en formation pour rallier un support de masse à des objectifs résolument émancipateurs. Inscrits dans le dépassement des clivages ethniques et des antagonismes sociaux, ils développent un discours unificateur autour des mots d'ordre magiques « indépendance », « démocratie », voire « socialisme». Comme tels, et dans le cadre des frontières aberrantes héritées de la colonisation, ils ont rempli la fonction de catalyseurs du nationalisme africain et ont été le véritable levier de l'indépendance. Le modèle du genre est le Convention People's Party fondé en 1949 en Gold Coast par Kwame Nkrumah, qui recourut à toutes les armes de l'agitation et de l'action légale, et qui eut de multiples émules en Afrique anglaise. Dans les possessions françaises, le congrès de Bamako réalise en 1946, sous le nom de Rassemblement démocratique africain (RDA), l'union de divers mouvements qui laisse en fait une large autonomie à leurs leaders respectifs. Initialement plus anticolonialiste qu'indépendantiste, le RDA a naturellement évolué au milieu des années 1950 vers la revendication à plus ou moins long terme de l'indépendance.

Face à cette brusque radicalisation, les puissances coloniales, au sortir de la guerre, se montrent réticentes. La thèse généralement admise, tant en France qu'en Angleterre, était celle de l'incapacité des autochtones à participer aux affaires politiques avant un long entraînement à la gestion des affaires locales. Acquis dès 1945 à l'indépendance de l'Inde, les travaillistes anglais entendaient agir en Afrique avec pragmatisme, par un jeu subtil de constitutions successives où l'Indirect Rule serait progressivement remplacé par le Responsible government 
puis par un self-government à même de représenter et d'impliquer toutes les composantes ethniques et religieuses de chaque territoire (thèse du multiracialisme). S'agissant des possessions françaises, et contrairement à ce qu'a pu accréditer un certain gaullisme complaisant, la conférence de Brazzaville (janvier-février 1944), simple réunion d'administrateurs coloniaux, ne préfigurait en rien une quelconque indépendance, ni même une simple autonomie. Elle se limitait à des réformes administratives et sociales, dénuées de tout prolongement politique. Dans le cadre étriqué et pseudo-fédéral de l'Union française, la Constitution de 1946 maintenait l'essentiel de la domination métropolitaine, même si, par le jeu de l'élection à l'Assemblée nationale et à l'Assemblée de l'Union française de plusieurs dizaines d'indigènes, et par la politique d'indigénisation amorcée par la loi-cadre Defferre de 1956, la IVe République s'est révélée, tout compte fait, une bonne école et un vivier de dirigeants africains.

Quant aux autres puissances coloniales, elles se montraient plus conservatrices encore et fort peu conscientes des mutations en cours. Le gouvernement belge restaura en 1945 le système paternaliste et autoritaire censé perpétuer un Congo docile et assisté, bien tenu par l'administration et les missions, au point de désavouer le plan Van Bilsen (1955), qui osait envisager l'indépendance dans un délai de trente ans, et suscitait la réaction indignée des milieux coloniaux. Quant au Portugal, la doctrine officielle de l'assimilation des indigènes était quotidiennement contredite par de multiples pratiques discriminatoires.

La décennie qui suit la Seconde Guerre mondiale est donc celle du raidissement des puissances coloniales, confortées dans leur immobilisme par la menace, réelle ou supposée, de la subversion communiste dans le contexte de la guerre froide. Toute perturbation de l'ordre politique ou social est, dès lors, impitoyablement réprimée, comme l'attestent les opérations de police qui s'échelonnent de 1947, à Madagascar, à la fin des années 1950, au Cameroun et au Kenya.

Le temps pourtant travaillait contre elles. Les excès mêmes de la répression renforçaient l'audience des mouvements contestataires et de leurs chefs, ces derniers retournant habilement contre le colonisateur les principes démocratiques dont se réclamaient officiellement les métropoles. Leur protestation trouvait aux Nations unies une tribune complaisante, à l'assemblée générale où l'Égypte de Nasser se fit leur 
meilleur avocat, mais aussi dans le groupe afro-asiatique formé en 1950 et au Comité de décolonisation fondé en 1961. Partagés entre leur anticolonialisme de principe et la priorité de la sécurité européenne, les États-Unis encourageaient discrètement les mouvements d'émancipation, dès lors qu'était levée l'hypothèque d'éventuelles sympathies communistes. L'Église catholique elle-même, dont les missions étaient pourtant étroitement liées à la domination coloniale, entendait préparer l'avenir. Les encycliques Evangelii Praecones (1951) et Fidei Dominum (1957) laissent entendre la contingence de la colonisation face à l'universalité de l'Église romaine, et ouvrent la voie à une précoce indigénisation de la hiérarchie ecclésiastique. À l'heure de la conférence de Bandoeng, qui accueillit plusieurs observateurs africains, et du camouflet subi à Suez par les puissances coloniales, il est clair que les jours du colonialisme étaient comptés.

Il n'est pas aisé de procéder à une périodisation pertinente de la décolonisation africaine, qui, de l'indépendance du Ghana en 1957 à celle de la Namibie en 1990, s'étend sur un tiers de siècle. En schématisant beaucoup, on retiendra trois phases.

La première couvre les années 1957-1960. Par son ampleur (une vingtaine d'États accède à l'indépendance) comme par son caractère négocié et relativement pacifique, elle laisse bien augurer de la transition coloniale à la souveraineté. C'est la Gold Coast qui donne le signal de l'émancipation, possession la plus riche et la plus évoluée de l'Afrique occidentale britannique. Le Convention People's Party a fait preuve de sa représentativité à la faveur de plusieurs élections et son leader Kwame Nkrumah, devenu Premier ministre, négocie loyalement l'indépendance de son pays, qui prend le nom de Ghana. Cette indépendance a un retentissement considérable, d'autant plus que Nkrumah l'exploite sans tarder par la réunion d'une conférence panafricaine à Accra en avril 1958. Elle accélère la revendication séparatiste de la Guinée française (où Sékou Touré fait, à cette fin, voter «non » à la Constitution française de 1958), et précipite au Congo belge la formation du Mouvement national congolais par Patrice Lumumba, parti indépendantiste et intertribal, principal interlocuteur des autorités belges et principal bénéficiaire (dans l'immédiat) d'une indépendance accordée dans la plus totale hâte le 30 juin 1960. À cette date, la Communauté française, entité plus ou moins confédérale 
imaginée par le général de Gaulle pour encadrer les liens de la France et de l'Afrique, a donné les signes évidents d'un épuisement précoce, tant en raison de l'exemple guinéen que de la perpétuation jugée insupportable par les États communautaires du centralisme métropolitain. Entre juin et octobre 1960, douze anciennes colonies (y compris Madagascar) et les deux territoires sous tutelle du Togo et du Cameroun accèdent à l'indépendance et signent des accords de coopération qui vont durablement les arrimer au pré carré français.

Une deuxième phase s'étend de 1960 à 1965, dominée par la décolonisation hésitante des possessions britanniques en Afrique occidentale (Nigeria), orientale (Tanganyika, Kenya, Ouganda) et centrale (Rhodésies). La bonne volonté du Premier ministre Macmillan et de son ministre Macleod, sensibles au Wind of the change, n'est pas en cause, mais plutôt les réticences du Colonial Office à reconnaître une indépendance jugée prématurée aux colonies à forte diversité ethnique ou religieuse (Nigeria) ou à fort peuplement blanc (Kenya, Rhodésies). D'où la conclusion de laborieux compromis et la perpétuation, jusqu'en 1963, d'une Fédération d'Afrique centrale fictivement fondée sur le multiracialisme, mais qui accordait aux Blancs une suprématie de fait. Les indépendances s'échelonnent de 1960 (Nigeria) à 1965 (Rhodésie du Nord, sous le nom de Zambie), et favorisent l'entrée de ces jeunes États dans le Commonwealth.

La proclamation unilatérale par le Premier ministre Ian Smith de l'indépendance de la Rhodésie du Sud, en novembre 1965, ouvre une troisième phase plus longue et plus conflictuelle, dominée par la résistance des «bastions blancs » (Afrique du Sud, Rhodésie du Sud, Portugal) à toute indépendance négociée. Il s'ensuit des conflits meurtriers qui juxtaposent, et dans une certaine mesure combinent, l'obstination du Portugal salazariste, en l'occurrence soutenu par l'Otan, à maintenir coûte que coûte les derniers vestiges d'un empire déchu, et celle des minorités blanches d'Afrique australe à institutionnaliser leur domination au mépris des injonctions de la communauté internationale. La « révolution des œillets » à Lisbonne, en avril 1974, met un terme à ces guerres ruineuses et permet aux possessions portugaises (Guinée-Bissau, Angola, Mozambique) d'accéder à l'indépendance l'année suivante, mais que vient perturber l'intervention des puissances étrangères mues par des intérêts économiques et des ambitions stratégiques. Il faut attendre 1980 pour que la guérilla menée par la Zanu 
de Robert Mugabe s'impose aux forces sud-rhodésiennes, et encore dix ans pour que l'écroulement de l'apartheid en Afrique du Sud amène la Namibie à l'indépendance.

Au terme d'un cycle plus que trentenaire, l'Afrique noire s'est donc vu reconnaître une souveraineté complète. La proclamation des indépendances constitue certes un aboutissement particulier à chaque territoire, mais certains traits comparables autorisent à esquisser une typologie des processus d'émancipation. Aucune indépendance ne fut accordée sans épreuve de force, à tout le moins sans tensions préalables qui ont traversé, on l'a vu, la décennie 1945-1955. On retiendra pourtant comme valable la distinction entre indépendances négociées (possessions françaises, anglaises et belges) et indépendances arrachées (colonies portugaises, Zimbabwe, Namibie). Encore faudrait-il distinguer dans le premier type les cas « exemplaires » d'une évolution institutionnelle (Union et Communauté françaises, Constitutions britanniques) et le cas du Congo belge, où l'indépendance fut négociée dans la plus totale improvisation. Et souligner aussi les limites d'une distinction qui ne préjuge en rien de l'évolution ultérieure des colonies émancipées.

Car le bilan de la décolonisation africaine n'est pas à la mesure des espoirs qu'elle avait suscités. L'instauration de régimes constitutionnels stables, d'inspiration parlementaire dans les anciennes possessions britanniques, présidentielle dans l'ancienne Communauté française, a été rapidement mise à mal par l'avènement du parti unique et la mue autocratique des pères fondateurs. La responsabilité en incombe sans doute aux puissances coloniales, par une formation insuffisante de cadres compétents, voire par une «politique des races » cultivant à dessein les différences ethniques. Mais il ne suffisait pas de procéder à une décolonisation toponymique (du Ghana en 1957 au Burkina-Faso en 1984, on relève une quinzaine d'appellations nouvelles) ni de parer tel ou tel pays d'une épithète présomptueuse (socialiste, populaire, démocratique...) pour garantir la cohésion nationale et la construction d'un État viable. Pléthorique et souvent corrompu, ce dernier est rapidement devenu l'enjeu de surenchères politiques ou de rivalités ethniques, et oscille de ce fait dangereusement entre l'instabilité chronique, le parasitisme bureaucratique et la confiscation clanique ou dictatoriale. 
La déposition et l'assassinat du président togolais Sylvanus Olympio, le 13 janvier 1963, a ouvert l'ère des coups d'État militaires. Sans avoir recherché systématiquement le pouvoir, l'armée est apparue comme la seule force de substitution à un pouvoir civil discrédité. Entre 1963 et 1965, on ne compte pas moins d'une trentaine de putschs frappant une vingtaine d'États, et entraînant parfois une intervention étrangère, celle de la France notamment, au Gabon ou au Tchad. Le drame vient de ce que, s'installant aux commandes, l'armée n'est pas mieux à même de résoudre les problèmes légués par le pouvoir civil ni d'opérer une quelconque moralisation de l'exercice du pouvoir. Un putsch en appelle souvent un autre, jusqu'à plonger certains pays dans une instabilité endémique et certaines dictatures militaires - Bokassa en République centrafricaine, Amin Dada en Ouganda, voire Mobutu au Zaïre - demeurent de sinistre mémoire.

Inspiré par Nkrumah, le regroupement des États africains s'était assigné pour tâches initiales l'accélération de la décolonisation et la lutte contre les menaces du néo-colonialisme, à plus long terme la recherche de l'indépendance économique et la promotion des solidarités de tous ordres du continent. Accra fut un temps la capitale du panafricanisme. Mais le radicalisme et l'autocratisme de Nkrumah, et l'échec plus général du « socialisme africain », levèrent des oppositions et des organisations concurrentes discrètement encouragées par l'étranger. La création la plus ambitieuse demeure l'Organisation de l'unité africaine, fondée à Addis-Abeba en 1963, dont la charte repose sur des principes acceptables pour tous: égalité et non-ingérence, règlement pacifique des conflits, intégrité territoriale des États dans le cadre des frontières héritées de la colonisation. Cette institutionnalisation du panafricanisme s'est révélée dans l'ensemble bien décevante. Sans doute a-t-elle poussé à l'accélération de l'émancipation des dernières colonies et permis d'arbitrer quelques conflits secondaires. Mais elle n'a pu intervenir efficacement dans les deux conflits majeurs qui ont déchiré l'Afrique post-coloniale - la guerre civile dans l'ancien Congo belge et la sécession biafraise au Nigeria -, pas plus qu'elle n'a maîtrisé les guerres intestines (Soudan, Liberia, Corne de l'Afrique, Burundi et Rwanda) ni les guerres inter-étatiques (entre Ouganda et Tanzanie, entre Sénégal et Mauritanie, entre le Zaïre et ses voisins) qui déchirent l'Afrique depuis trente ans. 
Au regard des potentialités de développement léguées par la décolonisation, il y a lieu d'être nuancé. Au seuil de l'indépendance, l'Afrique noire pouvait tirer parti de la valorisation économique entreprise par les puissances, et notamment du boom colonial de la décennie 1948-1958, ainsi que des infrastructures léguées par le colonisateur: ports, routes, voies ferrées, mais aussi aménagements hydrauliques et amorce d'industrialisation. Les Africains pourtant ne cessaient de dénoncer une mise en valeur orientée vers et pour la métropole, qu'il s'agisse de la préférence persistante donnée aux monocultures spéculatives ou des taux de profit exorbitants rapatriés. L'indépendance politique apparaissait dès lors comme le plus sûr moyen du développement et de l'élévation du niveau de vie, assortie d'une coopération financière et technique librement consentie.

À partir de là, les pays africains ont opté pour des politiques de développement divergentes. Certains se sont tournés vers un capitalisme largement ouvert aux capitaux étrangers (Côte-d'Ivoire, Gabon, Cameroun), d'autres s'en sont remis aux vertus supposées d'un socialisme étatique et nationalisateur (Ghana, Guinée, Congo), d'autres enfin ont cherché les voies d'un «socialisme africain » pragmatique et populaire (Tanzanie, Zambie).

De cette somme d'expériences, le bilan est assurément contrasté. D'indéniables progrès locaux ont été réalisés dans le domaine de l'industrialisation et de la diversification des ressources agricoles. Mais la décolonisation n'a nullement signifié la maîtrise du développement ni, a contrario, la rupture des liens de dépendance. La stratégie dite néocolonialiste des anciennes puissances coloniales et des grands groupes capitalistes nationaux ou multinationaux qui leur sont liés a été précocement dénoncée comme facteur de perpétuation de l'échange inégal. Celui-ci résulterait des effets combinés de la détérioration des termes de l'échange et du comportement prédateur, en capital et en travail, des investisseurs étrangers. D'où un déficit extérieur constant et un endettement chronique que l'aide étrangère aurait pour fonction essentielle d'éponger. L'échange inégal a donné lieu à de savantes controverses entre spécialistes. S'il s'appuie sur des hypothèses largement vérifiées, il ne saurait pourtant méconnaître ni les efforts déployés par les anciennes métropoles en vue de stabiliser le cours des matières premières agricoles et minières (accords de Lomé), ni le gâchis économique que 
représentent tant de travaux somptueux publics et privés entrepris après l'indépendance. Pour ne rien dire de l'incurie et de la corruption des cadres de l'appareil d'État dans la gestion d'une aide au départ généreusement octroyée. 